\title{
SOME PROPERTIES OF COMPOSITIONS AND THEIR APPLICATION TO THE BALLOT PROBLEM
}

\author{
S. G. Mohanty \\ (received July 27, 1964)
}

1. Introduction and summary. This paper is a continuation of two papers [4], [5] and brings out the solution of the ballot problem in its general form.

In [5], Narayana has considered a generalised occupancy problem which can be viewed as a problem in compositions of integers. In what follows, we use the definitions of [6]. Furthermore, we say that an $r$-composition $\left(t_{1}(m), \ldots, t_{r}(m)\right)$ of $m$ dominates an $r$-composition $\left(t_{1}(n), \ldots, t_{r}(n)\right)$ of $n$ $(m \geq n)$ if and only if

$$
\sum_{\alpha=1}^{i} t_{\alpha}(m) \geq \sum_{\alpha=1}^{i} t_{\alpha}(n), \quad \text { for } i=1, \ldots, r
$$

Evidently $\sum_{\alpha=1}^{r} \mathrm{t}_{\alpha}(\mathrm{m})=\mathrm{m}$ and $\sum_{\alpha=1}^{\mathrm{r}} \mathrm{t}_{\alpha}(\mathrm{n})=\mathrm{n}$. For integers $\mathrm{n}_{1}, \ldots, \mathrm{n}_{\mathrm{k}}$ such that $\mathrm{n}_{1} \geq \cdots \geq \mathrm{n}_{\mathrm{k}}$, we are required in [5] to determine the number of $r$-compositions of $n_{1}$ that dominate $r$-compositions of $\mathrm{n}_{2}$, that in turn dominate $r$-compositions of $n_{3}$, and so on. In other words, we are looking for the number of elements in the set $C=C\left(n_{1}, \ldots, n_{k} ; r\right)$ $=\left\{\left(t_{1}\left(n_{1}\right), \ldots, t_{r}\left(n_{1}\right)\right), \ldots,\left(t_{1}\left(n_{k}\right), \ldots, t_{r}\left(n_{k}\right)\right)\right.$ :

Canad. Math. Bull. vol. 8, no. 3, April 1965 


$$
\sum_{\alpha=1}^{i} t_{\alpha}\left(n_{j}\right) \geq \sum_{\alpha=1}^{i} t{ }_{\alpha}\left(n_{j+1}\right)
$$

for $i=1, \ldots, r$ and $j=1, \ldots, k-1\}$.

Now, corresponding to $C$, consider the set of lattice paths in a k-dimensional Euclidean space with axes $\mathrm{X}_{\ell}$ 's such that the $(k(i-1)+l)$ th segment $(i=1, \ldots, r$ and $\ell=1, \ldots, k)$ of any path is the distance $t_{i}\left(n_{\ell}\right)$ on $x_{\ell} \cdot$

Suppose a step in a path represents $k$ consecutive segments beginning from the segment on $X_{1}$. Then the set consists of paths from the origin to $\left(n_{1}, \ldots, n_{k}\right)$ not crossing the region bounded by $x_{k}=0, x_{j}=x_{j+1}, j=1, \ldots, k-1$ and having exactly $r$ components. Denote this set by $L *\left(n_{1}, \ldots, n_{k} ; r\right)$ or briefly L*. Thus the above construction has established a $1: 1$ correspondence between $C$ and $L *$ Letting $N\{$.$\} represent$ the number of elements in the set $\{$.$\} , it is shown in [5] that,$

$$
N\{C\}=N\{L * *\}=\left(n_{1}, \ldots, n_{k}\right)_{r}
$$

where

$$
\left(n_{1}, \ldots, n_{k}\right)^{\prime}=\left|\begin{array}{cccc}
\left(\begin{array}{c}
n_{1}-1 \\
r-1
\end{array}\right) & \left(\begin{array}{c}
n_{2}-1 \\
r
\end{array}\right) & \cdots & \left(\begin{array}{c}
n_{k}-1 \\
r+k-2
\end{array}\right) \\
\left(\begin{array}{c}
n_{1}-1 \\
r-2
\end{array}\right) & \left(\begin{array}{c}
n_{2}-1 \\
r-1
\end{array}\right) & \cdots & \left(\begin{array}{c}
n_{k}-1 \\
r+k-3
\end{array}\right) \\
\vdots & \vdots & \vdots & \vdots \\
\left(\begin{array}{c}
n_{1}-1 \\
r-k
\end{array}\right) & \left(\begin{array}{c}
n_{2}-1 \\
r-k+1
\end{array}\right) & \cdots & \left(\begin{array}{c}
n_{k}-1 \\
r-1
\end{array}\right)
\end{array}\right| .
$$

The determinant (4) plays an important role in this paper. It is also proved that $\left(n_{1}, \ldots, n_{k}\right)_{r}$ satisfies the following: 


$$
\begin{aligned}
& \mathrm{n}_{\mathrm{k}}-1 \quad \mathrm{n}_{\mathrm{k}-1}{ }^{-1} \quad \mathrm{n}_{1}{ }^{-1} \\
& \sum_{x_{k} \geq r}^{\sum} \sum_{x_{k-1} \geq x_{k}}^{\sum} \cdots \sum_{x_{1} \geq x_{2}}^{\Sigma}\left(x_{1}, \ldots, x_{k}\right)_{r}=\left(n_{1}, \ldots, n_{k}\right)_{r+1} .
\end{aligned}
$$

In section 2, we define a partial order on $\mathrm{C}$ and establish an isomorphism between $\mathrm{C}$ and a set of compositions of $M>n_{1}-r+k$, which is specified later. We also show that for $r=2$, it leads to an interesting correspondence among two different sets of lattice paths and a set of lattice points. As a special case, the number of $\mathrm{r}-\mathrm{compositions}$ of $\mathrm{n}$ that are [p]-dominated by a given $r$-composition of $m$ has been evaluated (for definition see [4] section 4). Section 3 deals with the application of results in section 2 , in order to provide a solution to a generalised class of the ballot problem [2, p. 66]. Finally, some identities which arise as a natural consequence of the above are included in the last section.

2. Isomorphism between two sets of compositions.

Recalling the definition of a composition vector [6] (that is, defining

$$
\left.\mathrm{T}_{i} *\left(\mathrm{n}_{\mathrm{j}}\right)=\sum_{\alpha=1}^{i} \mathrm{t}_{\alpha}\left(\mathrm{n}_{\mathrm{j}}\right)\right),
$$

it is remarked that the set $C$ is trivially in $1: 1$ correspondence with the set $T *=T *\left(n_{1}, \ldots, n_{k} ; r\right)=\left\{\left(T_{1} *\left(n_{1}\right), \ldots, T_{r}^{*}\left(n_{1}\right)\right), \ldots\right.$ $\left(\mathrm{T}_{1} *\left(\mathrm{n}_{\mathrm{k}}\right), \ldots, \mathrm{T}_{\mathrm{r}} *\left(\mathrm{n}_{\mathrm{k}}\right)\right)$ :

$$
T_{i} *\left(n_{j}\right) \geq T_{i}^{*}\left(n_{j+1}\right)
$$

for $i=1, \ldots, r$ and $j=1, \ldots, k-1\}$. Because of this correspondence and somewhat relative advantage of $T *$ over $C$, we refer frequently to $T *$ instead of $C$.

Definition: Given two elements $T_{1}^{*}=\left\{\left(T_{11} *\left(n_{1}\right), \ldots\right.\right.$, $\left.T_{r 1} *\left(n_{1}\right)\right), \ldots\left(T_{11} *\left(n_{k}\right), \ldots, T_{r 1} *\left(n_{k}\right)\right): T_{i 1} *\left(n_{j}\right) \geq T_{i 1} *\left(n_{j+1}\right)$ for all $i$ and $j\}$ and $T_{2} *=\left\{\left(T_{12} *\left(n_{1}\right), \ldots, T_{r 2} *\left(n_{1}\right)\right), \ldots\right.$,

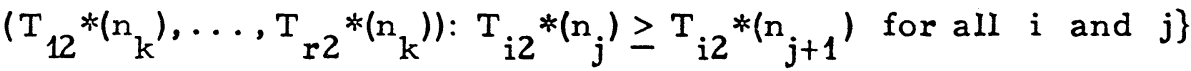


of $T *$, we say that $T_{1}^{*}$ dominates $T_{2} *$ if and only if

$$
T_{i 1} *\left(n_{j}\right) \geq T_{i 2} *\left(n_{j}\right) \text { for } i=1, \ldots, r \text { and } j=1, \ldots, k
$$

It can be shown that this relation is a partial order defined on elements of $T *$. Proceeding in a manner as in [6], we can also prove the following lemma.

LEMMA. The elements in $T$ * form a distributive lattice.

Next, it is easy to verify that the inequalities (6) for all i and $j$ are satisfied if and only if

(7) $T_{i+1} \geq T_{i j}$ for $i=1, \ldots, r-1$ and $j=1, \ldots, k$,

where $T_{i j}=T_{i} *\left(n_{j}\right)-i-j+k+1$ and $T_{r j}=n_{j}-r-j+k+1$. Since $T_{i k}<\ldots<T_{i 1}$ follows from (7), we now consider $k+1$ composition vectors $\left(T_{i k}, \ldots, T_{i 1}, M\right)$ for $i=1, \ldots, r$ where $M>n_{1}-r+k$ is a constant. Because of the inequalities in (7), we notice that $M>T_{i 1}$ for $i=1, \ldots, r$. Let $T=T\left(n_{1}, \ldots, n_{k} ; r\right)$ be the set

$$
\begin{aligned}
& \left\{\left(T_{r k}, \ldots, T_{r 1}, M\right), \ldots,\left(T_{1 k}, \ldots, T_{11}, M\right):\right. \\
& \left.T_{i+1 j} \geq T_{i j} \text { for } i=1, \ldots, r-1 \text { and } j=1, \ldots, k\right\} .
\end{aligned}
$$

In terms of compositions, it may be seen that $T$ is the set such that $(k+1)$-composition $\left(n_{k}-r+1, n_{k-1}-n_{k}+1, \ldots, n_{1}-n_{2}+1, M-n_{1}+r-k\right)$ of $M$ dominates $(k+1)$-compositions of $M$, each of which a gain dominates $(k+1)$-compositions of $M$ and so on. Using the simple transform $a_{i j}=T_{i} *\left(n_{j}\right)-i$, define the set $S=S\left(n_{1}, \ldots, n_{k} ; r\right)$ to be $\left\{\left(a_{r k}=n_{k}-r, \ldots, a_{r 1}=n_{1}-r\right), \ldots, a_{1 k}, \ldots, a_{11}\right)$ :

(8) $a_{i+1} \geq a_{i j}$ for $i=1, \ldots, r-1$ and $\left.j=1, \ldots, k\right\}$ where $\left(a_{i k}, \ldots, a_{i 1}\right)$ is a vector of non-negative, nondecreasing 
integers. The relation of domination on $T *$ through 1:1 transformations used above is extended to $T$ and $S$ and therefore we have the theorem.

THEOREM 1. Sets $T *, T$ and $S$ are isomorphic distributive lattices.

We have shown in section 1 that $T *\left(n_{1}, \ldots, n_{k} ; 2\right)$ through $C\left(n_{1}, \ldots, n_{k} ; 2\right)$ is $1: 1$ to $L *\left(n_{1}, \ldots, n_{k} ; 2\right)$. Also $T *\left(n_{1}, \ldots, n_{k} ; 2\right)$ is $1: 1$ to $S\left(n_{1}, \ldots, n_{k} ; 2\right)$ by the theorem. But $S\left(n_{1}, \ldots, n_{k} ; 2\right)$ is the set $\left\{\left(a_{k}, \ldots, a_{1}\right)\right\}$ of all vectors of non-negative and nondecreasing integers such that

$$
0 \leq a_{j} \leq n_{j}-2 \quad \text { for } j=1, \ldots, k
$$

Now, using the construction of lattice paths from non-negative nondecreasing vectors $[4$, p. 253], we notice that $\mathrm{S}\left(\mathrm{n}_{1}, \ldots, \mathrm{n}_{\mathrm{k}} ; 2\right)$ is $1: 1$ to the set $\mathrm{L}\left(\mathrm{n}_{1}, \ldots, \mathrm{n}_{\mathrm{k}}\right)$ of lattice paths from $(0,0)$ to $\left(n_{1}-1, k\right)$ not crossing the boundary given by the points $(0,0),,(1,1),\left(n_{1}-n_{2}+1,2\right),\left(n_{1}-n_{3}+1,3\right), \ldots,\left(n_{1}-n_{k}+1, k\right)$, Here we have two remarks to offer:

(a) The above lattice paths are equivalent to paths from $(0,0)$ to $\left(n_{i}, k\right)$ not touching the same boundary;

(b) The set of paths are, in general, also equivalent to paths from $(0,0)$ to $\left(n_{1}+e-2, k\right)$ [or $\left.\left(n_{1}+e-1, k\right)\right]$ not crossing [or not touching] the boundary $(0,0),(e, 1),\left(n_{1}-n_{2}+e, 2\right)$, $\left(n_{1}-n_{3}+e ; 3\right), \ldots,\left(n_{1}-n_{k}+e, k\right)$, where $e$ is a positive integer. From section 1,

$$
N\left\{L *\left(n_{1}, \ldots, n_{k} ; 2\right)\right\}=\left(n_{1}, \ldots, n_{k}\right)_{2} .
$$

We observe from (4) and (5) that 


$$
\begin{array}{cccc}
n_{k}-1 & n_{k-1}-1 & n_{1}-1 \\
x_{k}=1 & x_{k-1} \geq x_{k} & & x_{1} \geq x_{2}
\end{array} \quad 1=\left(n_{1}, \ldots, n_{k}\right)_{2} .
$$

The expression on the left hand side of (11) represents the number of lattice points in the region $R\left(n_{1}, \ldots, n_{k}\right)$ of k-dimensional Euclidean space bounded by hyperplanes

$$
\begin{aligned}
& x_{k}=\frac{1}{2}, x_{k}=n_{k}-\frac{1}{2}, x_{k-1}=x_{k}-\frac{1}{2}, x_{k-1}=n_{k-1}-\frac{1}{2}, . \\
& x_{1}=x_{2}-\frac{1}{2}, x_{1}=n_{1}-\frac{1}{2} .
\end{aligned}
$$

Thus, as a corollary of Theorem 1, we see that:

COROLLARY 1. $N\left\{L *\left(n_{1}, \ldots, n_{k} ; 2\right)\right\}=N\left\{L\left(n_{1}, \ldots, n_{k}\right)\right.$ $=N\left\{R\left(n_{1}, \ldots, n_{k}\right)\right\}=\left(n_{1}, \ldots, n_{k}\right)_{2}$.

It is not difficult to observe that $T\left(n_{1}, \ldots, n_{k} ; 2\right)$ represent the set of $(k+1)$-composition vectors $\left(T_{1}, \ldots, T_{k}, M\right)$ which are dominated by the $(k+1)$-composition vector

$$
\left(n_{k}-1, n_{k-1}, \ldots, n_{1}+k-2, M\right), \quad M>n_{1}+k-2 .
$$

Thus according to [6], the number $N\left\{T\left(n_{1}, \ldots, n_{k} ; 2\right)\right\}$ is give by $D_{k}$ in the recursive formula

$$
\left\{\begin{array}{c}
D_{0}=1 \\
D_{\mathrm{u}}=\sum_{\alpha=1}^{\mathrm{u}}(-1)^{\alpha+1}\left(\begin{array}{c}
\mathrm{n}_{\mathrm{k}-\mathrm{u}+\alpha}+\mathrm{u}-2 \\
\alpha
\end{array}\right) \mathrm{D}_{\mathrm{u}-\alpha} .
\end{array}\right.
$$

We know from Theorem 1 that

$$
N\left\{T\left(n_{1}, \ldots, n_{k} ; 2\right)\right\}=\left(n_{1}, \ldots, n_{k}\right)_{2} .
$$


Therefore, we have

COROLLARY 2. A solution of $\mathrm{D}_{\mathrm{u}}$ in (12) is $\left.\mathrm{n}_{\mathrm{k}-\mathrm{u}+1}, \mathrm{n}_{\mathrm{k}-\mathrm{u}+2}, \ldots, \mathrm{n}_{\mathrm{k}}\right)_{2}$.

A direct proof is also possible. We indicate it here. Using induction, $D_{u}$ can be written as the determinant

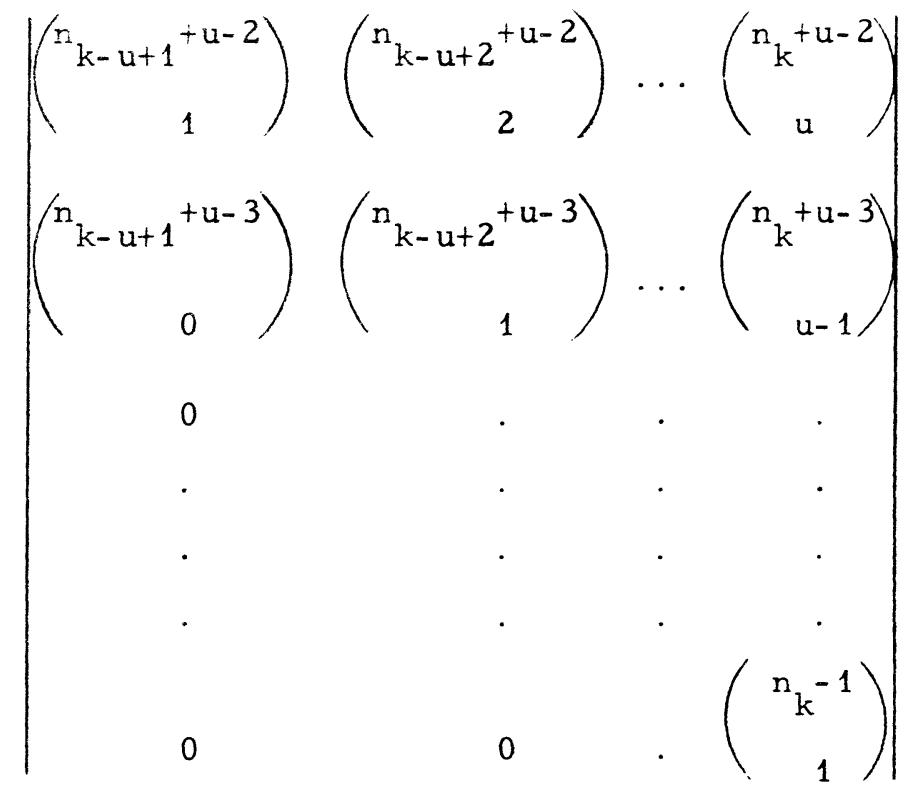

Subtraction of the $i^{\text {th }}$ row from the (i-1)st row $(i=2, \ldots, u)$, and repetition of this process reduces (13) to $\left(n_{k-u+1}, n_{k-u+2}, \ldots, n_{k}\right)$.

We now consider a problem, the solution of which is obtained with the help of Corollary 1 . It is required to determine the number of $r$-compositions of $n$ that are [s]-dominated by the $r$-composition $\left(t_{1}(m), \ldots, t_{r}(m)\right)$ of $m(m \geq s n)[4$, page 254]. The $r$-composition $\left(t_{1}(m), \ldots, t_{r}(m)\right)$ of $m[s]-$ dominates an $r$-composition $\left(t_{1}(n), \ldots, t_{r}(n)\right)$ of $n$ if and only if

$$
T_{i}(m) \geq s T_{i}(n)
$$


for $i=1, \ldots, r$. Inequalities (14) are equivalent to

$$
\left[\frac{T_{i}(m)}{s}\right] \geq T_{i}(n)
$$

for $i=1, \ldots, r$, where $[z]$ is the greatest integer less than or equal to $z$. Thus we are interested in the set of $r$-compositions of $n$ that are dominated by the $r$-composition

$$
\left(\left[\frac{T_{1}(m)}{s}\right],\left[\frac{T_{2}(m)}{s}\right]-\left[\frac{T_{1}(m)}{s}\right], \ldots,\left[\frac{m}{s}\right]-\left[\frac{T_{r-1}(m)}{s}\right]\right)
$$

of $\left[\frac{\mathrm{m}}{\mathrm{s}}\right]$. Transforming the set to the set of non-negative and non-decreasing vectors, as done earlier, we observe that the above set is $1: 1$ with the set of vectors $\left(a_{1}, \ldots, a_{r-1}\right)$ such that

(i) $a_{i}^{\prime} s$ are non-negative integers,

(ii) $a_{1} \leq \cdots \leq a_{r-1}$,

(iii) $0 \leq a_{i} \leq \min \left(\left[\frac{T_{i}(m)}{s}\right]-i, n-r\right)$ for $i=1, \ldots, r-1$

From the discussion following Theorem 1, we can get the number of such vectors, which is stated as a theorem.

THEOREM 2. The number of $r$-compositions of $n$ that are [s]-dominated by the $r$-composition $\left(t_{1}(m), \ldots, t_{r}(m)\right)$ of $\mathrm{m}(\mathrm{m} \geq \mathrm{sn})$ is

$$
\begin{aligned}
& \min \left(\left[\frac{\mathrm{T}-1}{\mathrm{~s}(\mathrm{~m})}\right]-\mathrm{r+1}, \mathrm{n}-\mathrm{r}\right)+2, \\
& \min \left(\left[\frac{\mathrm{T}-2^{(m)}}{\mathrm{s}}\right]-\mathrm{r}+2, \mathrm{n}-\mathrm{r}\right)+2, \ldots,
\end{aligned}
$$


$\min \left(\left[\frac{T_{1}(m)}{s}\right]-1, n-r+2\right)_{2}$.

3. Generalised ballot problems. The ballot problem $[2$, p. 66] and its extension have been discussed by several authors [1], [4], [7]. We state it as follows:

If in a ballot, Candidate $A$ scores $a$ votes and Candidate $B$ scores $b$ votes, where $a>b \mu, \mu$ being a positive integer, what is the probability that at each instant $A^{\prime}$ 's vote exceeds $\mu$ times $B^{\prime} s$ vote?

Representing each vote for $A$ by a unit horizontal step and each vote for $B$ by a unit vertical step, one of the solutions suggested in [4] uses the correspondence between lattice paths and non-negative non-decreasing vectors. In fact, the ballot problem with two candidates, in a generalised form, involves counting of lattice paths not touching a certain boundary which lies to the left of the paths. Recalling remarks (a) and (b), we have obtained the solution to such a problem in Section 2. In this context, we present below two theorems, the proof of which obviously follows from the preceeding results.

THEOREM 3. Let $\mathrm{x}$ and $\mathrm{y}$ respectively represent votes for $A$ and $B$ at a particular instant. Suppose that $A$ scores $a$ votes and $B$ scores $b$ votes such that $a>b \mu+\nu$, $\mu$ and $v$ being non-negative numbers. The number of ways in which $\mathrm{x}>\mathrm{y} \mu+\nu$ happens is given by

$$
(a-[\mu+v]+1, a-[2 \mu+v]+1, \ldots, a-[b \mu+v]+1)_{2} .
$$

At this point we note that Takács [7] gives a solution for general $\mu$ and $\nu=0$. When $\mu$ is a positive integer and $v=0$, the ballot problem reduces to the case stated at the beginning of this section. Therefore, the required number is $(a-\mu+1, \ldots, a-b \mu+1)_{2}=(a, b, \mu)$ say. We have to show that

$$
(a, b, \mu)=\frac{a-b \mu}{a+b}\left(\begin{array}{c}
a+b \\
b
\end{array}\right) \text {. }
$$

For $\mathrm{b}=1$, the result is true for all $\mathrm{a}$ and $\mu$. Adding 
each row to the previous row in the determinant $(a, b, \mu)$, we obtain

$$
(a, b, \mu)=(a+1, b, \mu)-(a+1, b-1, \mu)
$$

Hence

$$
\begin{aligned}
(a+1, b, \mu) & =(a+1, b-1, \mu)+(a, b, \mu) \\
& =(a+1, b-1, \mu)+(a, b-1, \mu)+(a-1, b, \mu) \\
& =(a+1, b-1, \mu)+(a, b-1, \mu)+\ldots+(b \mu+1, b-1, \mu),
\end{aligned}
$$

because $(b \mu, b, \mu)=0$. Applying induction, we get from (12) that

$$
\begin{aligned}
(a+1, b, \mu) & =\sum_{\alpha=b \mu+1}^{a+1}(\alpha, b-1, \mu)=\sum_{\alpha=b \mu+1}^{a+1} \frac{\alpha-(b-1) \mu}{\alpha+b-1}\left(\begin{array}{c}
\alpha+b-1 \\
b-1
\end{array}\right) \\
& =\sum_{\alpha=b \mu+1}^{a+1}\left(\begin{array}{c}
\alpha+b-2 \\
b-1
\end{array}\right)-\mu \sum_{\alpha=b \mu+1}^{a+1}\left(\begin{array}{c}
\alpha+b-2 \\
b-2
\end{array}\right) \\
& =\left(\begin{array}{c}
a+b \\
b
\end{array}\right)-\mu\left(\begin{array}{c}
a+b \\
b-1
\end{array}\right)=\frac{a-b \mu+1}{a+b+1}\left(\begin{array}{c}
a+b+1 \\
b
\end{array}\right),
\end{aligned}
$$

and the result follows.

Another variation of the ballot problem is given below and the result will be used in the next section.

THEOREM 4. For $A$ and $B$ having $a$ and $i$ vote: respectively, where $a>b_{1} \mu_{1}+v_{1}+\left(b-b_{1}\right) \mu_{2}+v_{2}, \mu_{1}, v_{2}, \psi_{2}$ and $v_{2}$ being non-negative numbers and $b_{1} \leq b$ a rormegrve integer, the number of ways in which $\mathrm{x}>\mathrm{y}_{\mu_{1}}+v_{1}$ when $0 \leq y \leq b_{1}$, and $x>b_{1} \mu_{1}+v_{1}+\left(y-b_{1}\right) \mu_{2}+v_{2}$ when $\mathrm{b}_{1} \leq \mathrm{y} \leq \mathrm{b}$ can happen is 


$$
\begin{gathered}
\left(a-\left[\mu_{1}+v_{1}\right]+1, \ldots, a-\left[b_{1} \mu_{1}+v_{1}\right]+1,\right. \\
a-\left[b_{1} \mu_{1}+v_{1}+\mu_{2}+v_{2}\right]+1, \ldots, \\
\left.a-\left[b_{1} \mu_{1}+v_{1}+\left(b-b_{1}\right) \mu_{2}+v_{2}\right]+1\right)_{2} .
\end{gathered}
$$

The above theorems illustrate the use of the results developed in Section 2, in some simple boundary cases.

4. Some combinatorial identities. The two A.P. case of [4, p. 256-258] is a special case of Theorem 4, with $\mu_{1}, v_{1}, \mu_{2}$ and $v_{2}$ as non-negative integers. Using the same notation as in [4], we therefore get

$$
\begin{aligned}
& N\left\{A_{p, q}(a+1, b+1 ; c+1, d+1)\right\}=N_{p, q}(a+1, b+1 ; c+1, d+1) \\
& =(a+(p-1) b+c+(q-1) d+2, a+(p-1) b+c+(q-2) d+2 ; \ldots, \\
& a+(p-1) b+c+2, a+(p-1) b+2, a+(p-2) b+2, \ldots, a+2) 2 \\
& =\sum_{k=0}^{q}(-1)^{k} \frac{a+1}{a+1+(p+q-k)(b+1)}\left(\begin{array}{c}
a+1+(p+q-k)(b+1) \\
p+q-k
\end{array}\right) . \\
& \frac{(q-k+1) b-c-(q-k) d}{(q-k+1) b-c-q d}\left(\begin{array}{c}
(q-k+1) b-c-q d \\
k
\end{array}\right)
\end{aligned}
$$

by Theorem 4 and Theorem 3 of [4]. Put b =1, c =1, d =0.

Then $A_{p, q}(a+1,2,2,1)$ is $1: 1$ with the set of paths from $(0,0)$ to $(p+a, p+q)$ not touching the line $x+q+1=y$, and the number of such paths is equal to

$$
\left(\begin{array}{c}
2 p+q+a \\
p+q
\end{array}\right)-\left(\begin{array}{c}
2 p+q+a \\
p-1
\end{array}\right)
$$

by [3]. Therefore, we have an identity 
(14)

$$
\begin{aligned}
& (\underbrace{p+2, \ldots, a+p}_{q}+2, a+p+1, a+p, \ldots, a+2)_{2} \\
& =\sum_{k=0}^{q}(-1)^{k} \frac{a+1}{a+1+2(p+q-k)}\left(\begin{array}{c}
a+1+2(p+q-k) \\
p+q-k
\end{array}\right)\left(\begin{array}{c}
q-k \\
k
\end{array}\right) \\
& =\left(\begin{array}{c}
2 p+q+a \\
p+q
\end{array}\right)-\left(\begin{array}{c}
2 p+q+a \\
p-1
\end{array}\right) .
\end{aligned}
$$

Consider $(p+a, p+q)$ as the origin, $x=p+a, y=p+q$ as $\mathrm{x}$-axis and $\mathrm{y}$-axis respectively, such that the old origin becomes $(p+q, p+a)$. Thus the previous set of paths is the same as the set of paths from $(0,0)$ to $(p+q, p+a)$ not touching $x+a+1=y$. The number in the latter set gives rise to the identity

$$
\begin{aligned}
& (\underbrace{p+q+2, \ldots, p+q}_{a}+2, p+q+1, \ldots, q+2)_{2} \\
& =\sum_{k=0}^{a}(-1)^{k} \frac{q+1}{q+1+2(p+a-k)}\left(\begin{array}{c}
q+1+2(p+a-k) \\
p+a-k
\end{array}\right)\left(\begin{array}{c}
a-k \\
k
\end{array}\right) \\
& =\left(\begin{array}{c}
2 p+q+a \\
p+a
\end{array}\right)-\left(\begin{array}{c}
2 p+q+a \\
p-1
\end{array}\right) .
\end{aligned}
$$

Either from the remark preceding (15) or from the obvious identity $\left(\begin{array}{c}2 p+q+a \\ p+q\end{array}\right)=\left(\begin{array}{c}2 p+q+a \\ p+a\end{array}\right)$, we see that (14) equals (15).

We can show that

$$
\begin{aligned}
& \sum_{k=0}^{p+q}(-1)^{k} \frac{a+1}{a+1+2(p+q-k)}\left(\begin{array}{c}
a+1+2(p+q-k) \\
p+q-k
\end{array}\right)\left(\begin{array}{c}
q-k \\
k
\end{array}\right) \\
& =\sum_{k=0}^{p+a}(-1)^{k} \frac{q+1}{q+1+2(p+a-k)}\left(\begin{array}{c}
q+1+2(p+a-k) \\
p+a-k
\end{array}\right)\left(\begin{array}{c}
a-k \\
k
\end{array}\right) \\
& =\left(\begin{array}{c}
2 p+q+a \\
p+q
\end{array}\right)=\left(\begin{array}{c}
2 p+q+a \\
p+a
\end{array}\right)
\end{aligned}
$$


by formula (17) in [9]. Therefore

$$
\text { (17) } \begin{aligned}
& \sum_{k=q+1}^{p+q}(-1)^{k} \frac{a+1}{a+1+2(p+q-k)}\left(\begin{array}{c}
a+1+2(p+q-k) \\
p+q-k
\end{array}\right)\left(\begin{array}{c}
q-k \\
k
\end{array}\right) \\
= & \sum_{k=a+1}^{p+a}(-1)^{k} \frac{q+1}{q+1+2(p+a-k)}\left(\begin{array}{c}
q+1+2(p+a-k) \\
p+a-k
\end{array}\right)\left(\begin{array}{c}
a-k \\
k
\end{array}\right) \\
= & \left(\begin{array}{c}
2 p+q+a \\
p-1
\end{array}\right) .
\end{aligned}
$$

Perhaps some of the identities might have been proved or can be proved directly. A less obvious identity arises as follows. In the ballot problem stated in Theorem 3 , set $v=0$ and $\mu=\frac{a}{b+1}$ where $a$ and $b+1$ are relatively prime numbers. Then an application of the result of Theorem 2 of [8] yields

$$
\begin{gathered}
\left(a-\left[\frac{a}{b+1}\right]+1, a-\left[\frac{2 a}{b+1}\right]+1, \ldots, a-\left[\frac{b a}{b+1}\right]+1\right)_{2} \\
=\frac{1}{a+b+1}\left(\begin{array}{c}
a+b+1 \\
a
\end{array}\right) .
\end{gathered}
$$

In conclusion, we remark that the solution in the form of a determinant might not reduce to a simpler expression, except in some special cases.

\section{REFERENCES}

1. A. Dvoretzky and Th. Motzkin, A Problem of Arrangements, Duke Mathematical Journal, XIV, (1947), p. 305.

2. W. Feller, An Introduction to Probability Theory and Its Applications, Second Edition, New York, John Wiley and Sons, Inc., 1957.

3. H. D. Grossman, Fun with Lattice Points, Scripta Mathematica, (1946), p. 224. 
4. S. G. Mohanty and T. V. Narayana, Some Properties of Compositions and their Applications to Probability and Statistics I, Biometrische Zeitschrift, Band 3, Heft 4, (1961), p. 252 .

5. T.V. Narayana, A combinatorial problem and its application to probability theory I, Journal of the Indian Society of Agricultural Statistics VII, (1955), p. 169.

6. T.V. Narayana and G. E. Fulton, A Note on the Compositions of an Integer, Canadian Mathematical Bulletin, Vol. I, (1958), p. 169.

7. L. Takács, A Generalisation of the Ballot Problem and Its Application in the Theory of Queues, Journal of American Statistical Association, Vol. 57, (1962), p. 327.

8. L. Takács, Ballot Problems, Z. Wahrscheinlichkeitstheorie, Vol. 1, (1962), p. 154.

9. H. W. Gould, Some Generalisations of Vandermonde's Convolution, American Mathematical Monthly, Vol. 63, (1956), p. 84 .

State University of New York

at Buffalo

and

McMaster University 